Check for updates

Cite this: Chem. Commun., 2020, 56,5405

Received 21st January 2020

Accepted 20th March 2020

DOI: $10.1039 / \mathrm{d} 0 \mathrm{cc} 00579 \mathrm{~g}$

rsc.li/chemcomm

\section{A mitochondria-targeting NIR fluorescent potassium ion sensor: real-time investigation of the mitochondrial $\mathrm{K}^{+}$regulation of apoptosis in situ $\dagger$}

\author{
Guangjie Song, ${ }^{\mathrm{ab}}$ Di Jiang, ${ }^{\mathrm{b}}$ Lei Wang, ${ }^{a}$ Juewei Ning, ${ }^{a}$ Xiangzhong Sun, ${ }^{a}$ \\ Fengyu Su, ${ }^{* c}$ Meiwan Chen ${ }^{* b}$ and Yanqing Tian (D) *a
}

The first NIR fluorescent mitochondria-targeting $\mathrm{K}^{+}$sensor, denoted as TAC-Rh, was developed. The produced sensor consists of a rhodamine analog as the fluorophore and triazacryptand (TAC) as the $\mathrm{K}^{+}$recognition unit. Compared to the $\mathrm{K}^{+}$sensors reported previously, TAC-Rh exhibits two unique optical properties: the largest Stokes shifts $(120 \mathrm{~nm})$ and the longest emission peak wavelength $(720 \mathrm{~nm})$. With the assistance of this novel sensor, real-time changes of $\mathrm{K}^{+}$concentrations in mitochondria during apoptosis were monitored for the first time. Moreover, it was also the first time that the relationship between mitochondrial $\mathrm{K}^{+}$flux and apoptosis was investigated in real time using fluorescence imaging.

The potassium ion $\left(\mathrm{K}^{+}\right)$is one of the predominant ions in living cells (about $150 \mathrm{mM}$ ), and is closely involved in many biological processes, such as kidney function, nerve transmission, heart beat and muscle contraction. ${ }^{1}$ Yu et al. first proposed an association between apoptosis and the loss of intracellular potassium ions. ${ }^{2}$ In 2016, Vodnala et al. discovered that an increase in the concentration of $\mathrm{K}^{+}$in tumor microenvironments could reduce the activity of $\mathrm{T}$ cells and prevent their anti-tumor function, ${ }^{3}$ and discovered in 2019 that an increased concentration of $\mathrm{K}^{+}$would also destroy $\mathrm{T}$ cell metabolism and nutrient uptake, leading to a starvation state known as autophagy. ${ }^{4}$ These discoveries opened up new avenues for developing therapies aimed at mobilizing the immune system against cancer, with these efforts involving exploring the role of $\mathrm{K}^{+}$in these processes. Therefore, determination of intracellular potassium levels is essential.

More and more studies have found mitochondria to be involved in a variety of key events involving the regulation of

\footnotetext{
${ }^{a}$ Department of Materials Science and Engineering Southern University of Science and Technology, Shenzhen, 518055, China. E-mail: tianyq@sustech.edu.cn

${ }^{b}$ State Key Laboratory of Quality Research in Chinese Medicine Institute of Chinese Medical Sciences, University of Macau, Macao 999078, China.

E-mail:mwchen@umac.mo

${ }^{c}$ Academy for Advanced Interdisciplinary Studies Southern University of Science and Technology, Shenzhen, 518055, China. E-mail: fysu@sustech.edu.cn

$\dagger$ Electronic supplementary information (ESI) available: Synthesis, additional methods, and figures (Fig. S1-S21). See DOI: 10.1039/d0cc00579g
}

apoptosis. ${ }^{5,6}$ However, most of these studies did not delve deeply into the organelle level to explore the relationship between mitochondrial $\mathrm{K}^{+}$and apoptosis due to the lack of appropriate tools. Therefore, constructing a tool to trace in real time the flow of $\mathrm{K}^{+}$in mitochondria would be expected to be very beneficial for analyzing the relationship between mitochondrial $\mathrm{K}^{+}$and apoptosis.

In previous measurements of mitochondrial $\mathrm{K}^{+}$concentration, it was usually necessary to isolate mitochondria, a complex process not feasible for determining the concentration of mitochondrial $\mathrm{K}^{+}$ in real time. ${ }^{7}$ Confocal laser scanning microscopy has turned out to be an important tool for understanding the biological states of metal ions, and doing so in a damage-free manner with high timespace resolution. ${ }^{8}$ However, currently, only a few $\mathrm{K}^{+}$sensors have been prepared. ${ }^{9}$ Moreover, PBFI, the most common potassium indicator, was reported to be interfered with by sodium to some extent. ${ }^{10}$ Since $\mathrm{He}$ et al. first reported a $\mathrm{K}^{+}$sensor using a highly selective triazacryptand (TAC) ligand as the $\mathrm{K}^{+}$-sensing moiety, ${ }^{11} \mathrm{a}$ few TAC-derived $\mathrm{K}^{+}$sensors were further developed and used for intracellular imaging of $\mathrm{K}^{+} .9 c, e, g, 12$

The emissions of most fluorescent sensors have been observed to occur in the ultraviolet visible (UV/Vis) range $(400-700 \mathrm{~nm}$ in wavelength), leading to light-induced toxicity, and weak tissue penetration and resolution, when applied in vivo due to the auto fluorescence and light absorption of biomolecules. It would be preferable to pursue low-energy NIR fluorescent sensors to minimize light-induced toxicity, to lower the level of interference from biomolecules, and deepen tissue penetration..$^{13}$ However, the fluorophores used for $\mathrm{K}^{+}$sensors, including boron dipyrromethenes (BODIPYs), ${ }^{9 f, 14}$ naphthalimides, ${ }^{9,, 15}$ and other dyes ${ }^{9 b, 16}$ have emission wavelengths below $600 \mathrm{~nm}$. So far, only four $\mathrm{K}^{+}$fluorescent sensors with emission wavelengths of over $600 \mathrm{~nm}$ have been reported (Zhou et al., 2011, $\lambda_{\max e m}=650 \mathrm{~nm} ;{ }^{9 c}$ Sui et al., 2015, $\lambda_{\text {max em }}=650 \mathrm{~nm} ;{ }^{9 e}$ Müller et al., 2016, $\lambda_{\max \text { em }}=688 \mathrm{~nm} ;{ }^{9 d}$ Bandara et al., 2017, $\lambda_{\operatorname{maxem}}=680 \mathrm{~nm}^{17}$ ) (see Table S1 in ESI†). Although these fluorescence emission wavelengths are partly situated in the NIR region (700-900 $\mathrm{nm}$ in wavelength), the peaks of all these fluorescence emissions are at wavelengths of shorter than $700 \mathrm{~nm}$. 
Ideal intracellular $\mathrm{K}^{+}$sensors should display the following characteristics: ${ }^{18}$ long-wavelength fluorescence, wide dynamic $\mathrm{K}^{+}$detection range (100-300 $\mathrm{mM}$ ), insensitivity to $\mathrm{Na}^{+}(5-15 \mathrm{mM}$ in intracellular fluid) and other metal ions at physiological concentrations, $\mathrm{pH}$ insensitivity, and rapid response. Our strategy was to integrate TAC for $\mathrm{K}^{+}$recognition with a rhodamine analog as an NIR fluorophore. Herein, TAC-Rh with a $\mathrm{K}^{+}$detection range of 16 to $400 \mathrm{mM}$ was designed and synthesized, enabling its suitable application for sensing intracellular $\mathrm{K}^{+}$. TAC-Rh is the first NIR fluorescent $\mathrm{K}^{+}$sensor with a peak of emission wavelength of up to $720 \mathrm{~nm}$, a value greater than those of all fluorescent $\mathrm{K}^{+}$sensors reported to date. And TAC-Rh in HEPES buffer shows a Stokes shift of $120 \mathrm{~nm}$, also a value larger than those of all reported potassium ion sensors. In addition, TAC-Rh with its positive charge preferentially accumulated in mitochondria, realizing in situ monitoring of mitochondrial $\mathrm{K}^{+}$concentration. To date, only two mitochondrion-targeting $\mathrm{K}^{+}$sensors with short emission wavelengths have been reported by us. ${ }^{9 h, 12}$ And the sensors were only used to observe the fluxes of $\mathrm{K}^{+}$in mitochondria, and were not used for exploring mitochondrial $\mathrm{K}^{+}$regulation of apoptosis. In this work, the optical properties of TAC-Rh and its applications in studying the relationship between mitochondrial potassium concentration and apoptosis were investigated.

Fig. 1a shows the synthetic route to TAC-Rh. In brief, TAC-Rh was obtained by forming a covalent $\mathrm{C}=\mathrm{C}$ bonds between TAC and the rhodamine analog, in turn achieved by carrying out a condensation reaction of TAC-CHO ${ }^{9 c}$ with compound $\mathbf{1}$ in an acetic anhydride solution. And the structure of TAC-Rh was characterized by performing ${ }^{1} \mathrm{H}$ NMR spectroscopy and highresolution mass spectroscopy (Fig. S1 and S2, ESI $\dagger$ ).

Due to the electrostatic interaction resulting from the positively charged cationic TAC-Rh and negative transmembrane potential of mitochondria, most of the TAC-Rh was expected to localize in the mitochondria. ${ }^{19}$ In the structure of the TAC-Rh molecule,

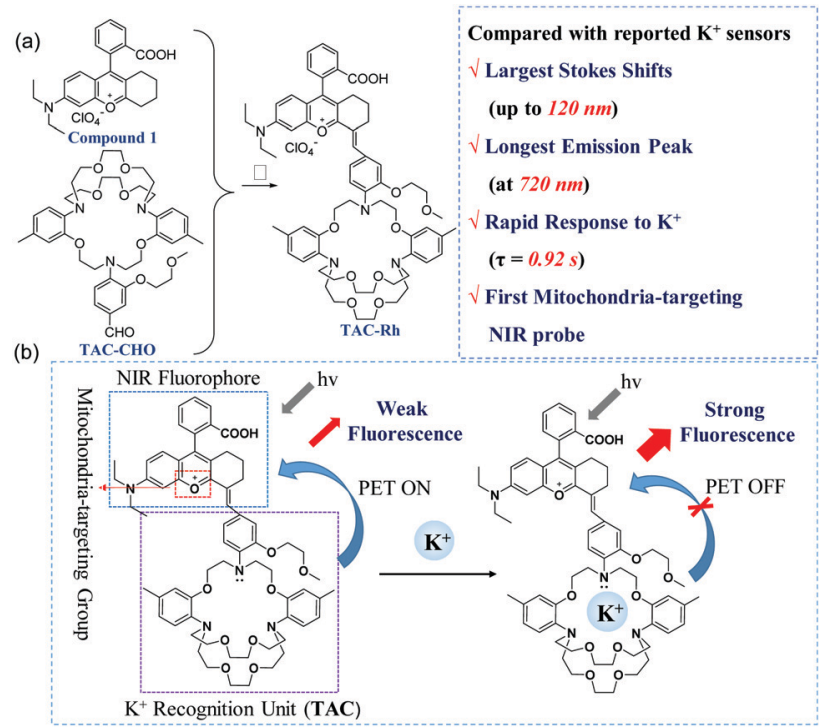

Fig. 1 Rational design, synthetic route (a), and proposed PET process (b) for TAC-Rh; I: acetic anhydride, $90^{\circ} \mathrm{C}, 8 \mathrm{~h}$. the tertiary amino group of TAC acted as an electron donor and the rhodamine analog fluorophore served as an electron acceptor. The fluorescence quenching effect took place due to the photoinduced electron transfer (PET) (Fig. 1b) from TAC to the rhodamine analog. As a result, TAC-Rh in free form displayed weak emission, while a significant fluorescence intensity increase occurred upon the addition of $\mathrm{K}^{+}$because the complexation between TAC and $\mathrm{K}^{+}$effectively inhibited the fluorescence quenching from PET. Absorption and fluorescence titration experiments of TAC-Rh in a HEPES buffer containing centrimonium bromide (CTAB) as a surfactant, which can increase the solubility of the probe, were performed at various $\mathrm{K}^{+}$concentrations. The acquired UV spectra of the TAC-Rh- $\mathbf{K}^{+}$complex show a stronger UV absorption than that of the free TAC-Rh after the addition of $\mathrm{K}^{+}$(Fig. S3, ESI $\dagger$ ). As exhibited in Fig. 2a and b, a remarkable fluorescence response at $720 \mathrm{~nm}$ was observed with an excitation wavelength of $600 \mathrm{~nm}$, and the fluorescence response of TAC-Rh showed a linear relationship to $\log \left[\mathrm{K}^{+}\right]$in the range $16-400 \mathrm{mM}$. The $K_{\mathrm{d}}$ value was determined using the Benesi-Hildebrand plot to be $105 \mathrm{mM}$ (Fig. S3b, ESI $\dagger$ ), ${ }^{9 c}$ indicating the suitability of using TAC-Rh for monitoring intracellular $\mathrm{K}^{+}$levels.

Any intracellular $\mathrm{K}^{+}$sensor should be designed to be insensitive to other metal ions at their intracellular physiological levels. Therefore, the fluorescence changes for TAC-Rh were tested in the presence of the following intracellular cations at their respective physiological concentrations: $\mathrm{Cu}^{2+}(50 \mu \mathrm{M}), \mathrm{Ca}^{2+}$ (2 mM), $\mathrm{Mg}^{2+}(2 \mathrm{mM}), \mathrm{Mn}^{2+}(50 \mu \mathrm{M}), \mathrm{Fe}^{3+}(50 \mu \mathrm{M}), \mathrm{Fe}^{2+}(50 \mu \mathrm{M})$,
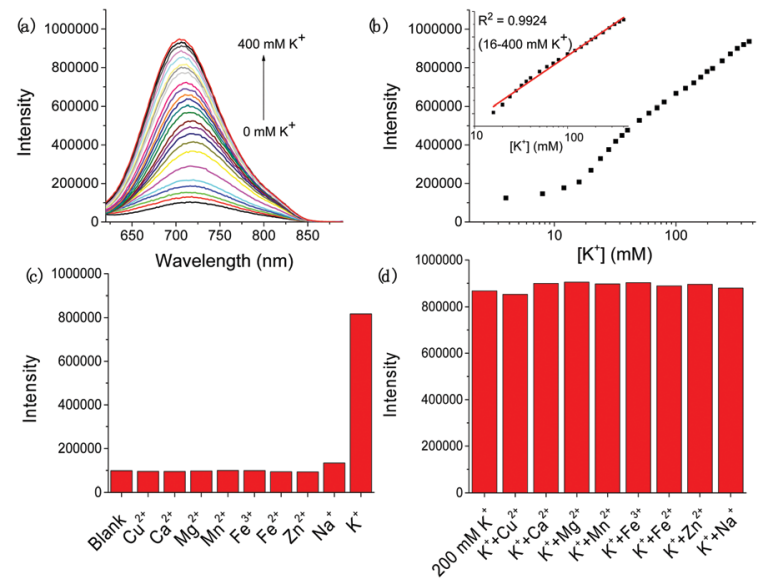

Fig. 2 Fluorescence titration spectra produced by gradually adding $\mathrm{KCl}$ at concentrations from 0 to $400 \mathrm{mM}$ to a solution of TAC-Rh $(10 \mu \mathrm{M})$ in HEPES/ $/ \mathrm{Cl}$ buffer $(\mathrm{pH} 7.4,5.0 \mathrm{mM}) / \mathrm{CTAB}(0.5 \mathrm{mM})$ and exciting the solutions with light of a wavelength of $600 \mathrm{~nm}$ (a). Plot of the fluorescence intensity of TAC-Rh at $720 \mathrm{~nm}$ versus $\mathrm{K}^{+}$concentration. The inset shows the relationship between the fluorescence intensity and $\mathrm{K}^{+}$concentration at concentrations between 16 and $400 \mathrm{mM}$ and a fit of a line to these data $\left(R^{2}=0.9924\right)$ (b). Fluorescence intensities at $720 \mathrm{~nm}$ of various solutions of TAC-Rh $(10 \mu \mathrm{M})$ containing different metal cations in HEPES buffer $(\mathrm{pH} 7.4$, $5.0 \mathrm{mM}) / C T A B(0.5 \mathrm{mM})$ (c). Fluorescence intensities at $720 \mathrm{~nm}$ of various solutions of the TAC-Rh- $\mathrm{K}^{+}(200 \mathrm{mM})$ complex containing other metal cations. The ions were from $\mathrm{CuCl}_{2}(50 \mu \mathrm{M}), \mathrm{CaCl}_{2}(2 \mathrm{mM}), \mathrm{MgCl}_{2}(2 \mathrm{mM})$, $\mathrm{MnCl}_{2}(50 \mu \mathrm{M}), \mathrm{FeCl}_{3}(50 \mu \mathrm{M}), \mathrm{FeCl}_{2}(50 \mu \mathrm{M}), \mathrm{ZnCl}_{2}(2 \mathrm{mM}), \mathrm{NaCl}(15 \mathrm{mM})$, and $\mathrm{KCl}(200 \mathrm{mM})$, i.e., all of them at their respective intracellular physiological concentrations (d). 
$\mathrm{Zn}^{2+}(2 \mathrm{mM}), \mathrm{Na}^{+}(15 \mathrm{mM})$, and $\mathrm{K}^{+}(150 \mathrm{mM})$. The results indicated that TAC-Rh showed little response to any of the other intracellular cations (Fig. 2c), and it responded to $\mathrm{K}^{+}$ without interference in the presence of any of the above cations (Fig. 2d). The fluorescence intensities of TAC-Rh and TAC-Rh$\mathrm{K}^{+}$were also insensitive to $\mathrm{pH}$ over the range $\mathrm{pH}$ 5.0-9.0 (Fig. S4, ESI $\dagger$ ). Notably, a remarkable fluorescence turn-on response was observed almost immediately after adding of $\mathrm{K}^{+}$, revealing the rapid response of TAC-Rh to $\mathrm{K}^{+}\left(\tau_{95 \%}=0.92 \mathrm{~s}\right.$ ) (Fig. S5, ESI $\dagger$ ).

Based on the above satisfactory photophysical properties of TAC-Rh in sensing $\mathrm{K}^{+}$, it was further applied to track potassium fluxes in live cells (HeLa cells, MCF7 cells, and MDA-MB-231 cells) by recording fluorescence intensities in real time. The cytotoxicity of TAC-Rh towards HeLa, MCF7 and MDA-MB-231 cells was first assessed by performing the 3-(4,5-dimethyl-2thiazolyl)-2,5-diphenyl-2- $H$-tetrazolium bromide (thiazolyl blue tetrazolium bromide, MTT)-based colorimetric assay. After being incubated with TAC-Rh at a concentration of $3 \mu \mathrm{M}$ for $2 \mathrm{~h}$, the viability of each of the three cell lines was over 95\% (Fig. S8, ESI $\dagger$ ), suggesting that TAC-Rh had no marked cytotoxicity toward the three cell lines under these experimental conditions.

The ability of TAC-Rh to target mitochondria was then assessed by evaluating the colocalization of TAC-Rh using MitoTracker Green (MTG, a commercial mitochondrial marker). The results showed that TAC-Rh mainly localized in mitochondria with a Mander's overlap co-efficiency of 0.94 (Fig. 3).

After the demonstration of the specific colocalization of TAC-Rh in mitochondria, TAC-Rh was used to monitor mitochondrial $\mathrm{K}^{+}$fluxes. Three kinds of cells (HeLa, MCF7 and MDA-MB-231 cells) internalized with TAC-Rh $(3 \mu \mathrm{M})$ for $30 \mathrm{~min}$ were treated with ionomycin or nigericin, which can effectively induce $\mathrm{K}^{+}$efflux. The intensity of the fluorescence of TAC-Rh in the single ionomycin or nigericin groups significantly decreased within $2 \mathrm{~min}$, as did the rate of efflux of mitochondrial $\mathrm{K}^{+}$ (Fig. S12-S19, ESI $\dagger$ ). While as a control, fluorescence signals in all three of these cells without any treatment showed relatively small decreases in 10 min (Fig. S9-S11, ESI $\dagger$ ). Furthermore, mitochondrial $\mathrm{K}^{+}$effluxes could be obviously delayed by treating the cells with ionomycin/nigericin in an extracellular environment having a high concentration of $\mathrm{K}^{+}$, which was supposed to have an inhibitory effect on potassium ion efflux owing to the decreased $\left[\mathrm{K}^{+}\right]$gradient between the insides and outsides of the

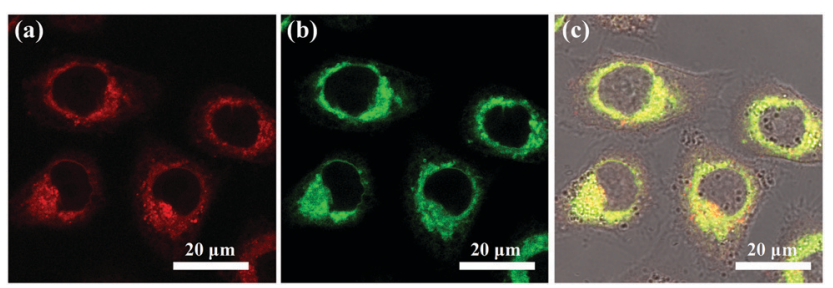

Fig. 3 Confocal fluorescence microscope images of HeLa cells co-stained with MitoTracker Green FM and TAC-Rh (3 $\mu \mathrm{M})$. Red emission from TAC-Rh (a). Green emission from MitoTracker Green FM (b). Overlay of MitoTracker Green and TAC-Rh (c). Excitation filter: $638 \mathrm{~nm}$ for TAC-Rh, $488 \mathrm{~nm}$ for Mito-Tracker Green. Emission: 650-750 nm for TAC-Rh, 500-550 nm for Mito-Tracker Green. mitochondria. Delayed mitochondrial $\mathrm{K}^{+}$efflux was observed in the ionomycin- or nigericin-treated groups when the cells in culture medium containing high concentrations of $\mathrm{K}^{+}$compared those containing a normal concentration of $\mathrm{K}^{+}$(Fig. S12-S19, ESI $\dagger$ ). The above phenomenon indicated that TAC-Rh can respond in real time to mitochondrial $\mathrm{K}^{+}$levels via fluorescence intensity changes.

D'Mello et al. pointed out that the depletion of potassium ions induced by ionomycin in cells could lead to cell shrinkage, activation of caspases, and DNA breakage, and finally cell apoptosis. $^{20}$ In this work, besides the observation of ionomycininduced mitochondrial $\mathrm{K}^{+}$efflux by the designed sensor TAC-Rh, annexin V/FITC-PI staining was simultaneously performed to in situ monitor the apoptosis process. The results showed that along with the markedly decreased fluorescence intensity of TACRh within 10 min of treatment with ionomycin (Fig. 4a and c), the fluorescence signal of annexin V/FITC indicating early-stage apoptosis gradually strengthened. And the typical characteristics of cell apoptosis including cell shrinkage and membrane blebbing were observed in the bright field, but the positive PI signals indicating late-stage apoptosis or dead cells did not appear until $\sim 30 \mathrm{~min}$ of treatment with ionomycin. The results indicated that mitochondrial $\mathrm{K}^{+}$efflux and early-stage apoptosis occurred almost simultaneously in the presence of ionomycin. In contrast, cells in control groups without drug stimulation only showed a small decrease in fluorescence intensity within $30 \mathrm{~min}$, and no significant morphological changes were observed (Fig. 4a and b). According to these results, we can infer that a potential correlation may exist between mitochondrial $\mathrm{K}^{+}$efflux and early-stage apoptosis.

Furthermore, while a reactive oxygen species (ROS)-induced apoptosis has been indicated to trigger a large amount of potassium ions flowing out of the cytoplasm, ${ }^{21}$ levels of $\mathrm{K}^{+}$in mitochondria during apoptosis have remained undetermined in real time owing to the lack of organelle-specific $\mathrm{K}^{+}$sensors. Since TAC-Rh was determined to be an excellent mitochondriatargeting fluorescent $\mathrm{K}^{+}$sensor, we further applied it to realtime monitoring of mitochondrial $\mathrm{K}^{+}$changes in the $\mathrm{H}_{2} \mathrm{O}_{2}$-induced apoptotic model. The results showed that compared with the control group (without $\mathrm{H}_{2} \mathrm{O}_{2}$ ), the cells of the $\mathrm{H}_{2} \mathrm{O}_{2}$-treated group were shriveled and the green fluorescence of FITC generally increased; meanwhile, the intensity of the overall red fluorescence of TAC-Rh declined gradually, indicating the ability of the apoptosis induced by $\mathrm{H}_{2} \mathrm{O}_{2}$ to trigger mitochondrial potassium ion efflux (Fig. S20, ESI $\dagger$ ). This was the first time that mitochondrial $\mathrm{K}^{+}$has been imaged in real time during the process of apoptosis.

As a high concentration of extracellular $\mathrm{K}^{+}$can reduce apoptosis and prolong survival of some cells by decreasing the $\left[\mathrm{K}^{+}\right]$gradient between the inside and outside of cells, ${ }^{22}$ achieving a delayed apoptosis by increasing the concentration of extracellular $\mathrm{K}^{+}$ was further carried out. As can be seen in Fig. S21 (ESI $\dagger$ ), in contrast to the noticeable weakening of the fluorescence of TAC-Rh and the obvious strengthening of FITC signals in the normal culture medium within 8 min of ionomycin stimulation, negligible small changes were observed in the red fluorescence of TAC-Rh and the green fluorescence of FITC as well as in the 

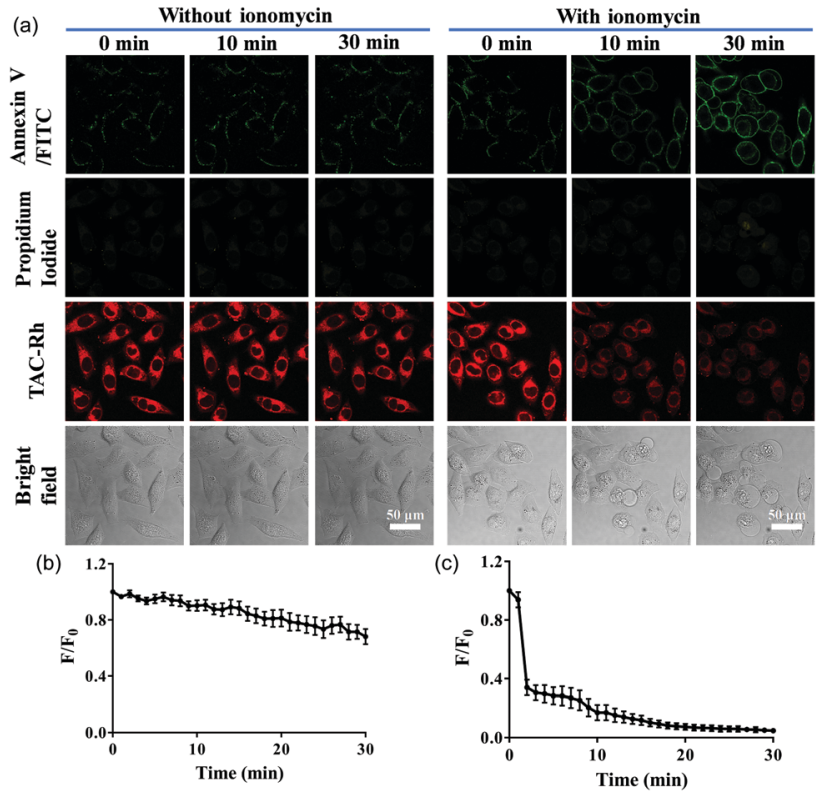

Fig. 4 Time-dependent confocal fluorescence microscopy images of HeLa cells stimulated without and with ionomycin (20 mM) into culture medium stained with annexin V/FITC, propidium lodide and TAC-Rh (a). Average fluorescence intensity ratio (i.e., $F / F_{0}$ ) values of TAC-Rh without ionomycin (b) and with ionomycin (c) as measured using Image $\mathrm{J}$, with $F_{0}$ denoting the average fluorescence intensity at $t=0 \mathrm{~min}$, and $F$ the average fluorescence intensity at a given time point. Excitation filter: $488 \mathrm{~nm}$ for annexin V/FITC, $552 \mathrm{~nm}$ for PI, $638 \mathrm{~nm}$ for TAC-Rh; emission: $493-540 \mathrm{~nm}$ for annexin V/FITC, $600-650 \mathrm{~nm}$ for PI, $650-750 \mathrm{~nm}$ for TAC-Rh

cell morphology in medium containing $80 \mathrm{mM} \mathrm{KCl}$. These phenomena demonstrated that high concentrations of extracellular $\mathrm{K}^{+}$could simultaneously inhibit the outflow of $\mathrm{K}^{+}$from mitochondria as well as early-stage apoptosis.

In summary, we have developed the first mitochondria-targeting NIR fluorescence $\mathrm{K}^{+}$sensor (TAC-Rh) by integrating a NIR rhodamine analog with the $\mathrm{K}^{+}$-binding group TAC. The fluorescence intensity of TAC-Rh showed an excellent $\left[\mathrm{K}^{+}\right]$-dependent response and a linear relationship with $\log \left[\mathrm{K}^{+}\right]$in the $\left[\mathrm{K}^{+}\right]$range $16-400 \mathrm{mM}$, features suitable for detecting changes in mitochondria $\mathrm{K}^{+}$concentration. The large Stokes shift and NIR emission of TAC-Rh minimized the photo-bleaching, light-induced injury, and interference by hemoglobin oxygenation and the cellular fluorescence background. Due to these excellent performance measures, TAC-Rh was found to be an excellent material to explore mutual regulation between mitochondrial $\mathrm{K}^{+}$flux and apoptosis. This study indicated that (1) HeLa cells treated with ionomycin could result in excessive mitochondrial $\mathrm{K}^{+}$efflux accompanied by apoptosis, (2) $\mathrm{H}_{2} \mathrm{O}_{2}$-induced apoptosis would trigger a large amount of $\mathrm{K}^{+}$ flux out of mitochondria, and (3) ionomycin-induced mitochondrial $\mathrm{K}^{+}$efflux and apoptosis could be inhibited by increasing the $\mathrm{K}^{+}$ concentration in culture medium. Therefore, TAC-Rh was shown to offer a novel strategy to understand mitochondrial $\mathrm{K}^{+}$changes during apoptosis, and further investigations using TAC-Rh would help researchers derive more information about apoptosis.

The authors would like to thank the National Natural Science Foundation of China (21774054, 21574061), the Shenzhen fundamental research programs (JCYJ20170412152922553), the Macao Science and Technology Development Fund (083/ 2017/A2), and the Research Fund of the University of Macau (MYRG2016-00130-ICMS-QRCM, MYRG2017-00182-ICMS).

\section{Conflicts of interest}

There are no conflicts to declare.

\section{Notes and references}

1 C. M. Lopez, A. E. Pineiro, N. Nunez, A. M. Avagnina, E. C. Villaamil and O. E. Roses, Pharmacol. Res., 2000, 42, 599.

2 S. P. Yu, C. H. Yeh, S. L. Sensi, B. J. Gwag, L. M. Canzoniero, Z. S. Farhangrazi, H. S. Ying, M. Tian, L. L. Dugan and D. W. Choi, Science, 1997, 278, 114.

3 R. Eil, S. K. Vodnala, D. Clever, C. A. Klebanoff, M. Sukumar, J. H. Pan, D. C. Palmer, A. Gros, T. N. Yamamoto, S. J. Patel, G. C. Guittard, Z. Yu, V. Carbonaro, K. Okkenhaug, D. S. Schrump, W. M. Linehan, R. Roychoudhuri and N. P. Restifo, Nature, 2016, 537, 539.

4 S. K. Vodnala, R. Eil, R. J. Kishton, M. Sukumar, T. N. Yamamoto, N. H. Ha, P. H. Lee, M. Shin, S. J. Patel, Z. Yu, D. C. Palmer, M. J. Kruhlak, X. Liu, J. W. Locasale, J. Huang, R. Roychoudhuri, T. Finkel, C. A. Klebanoff and N. P. Restifo, Science, 2019, 363, 135.

5 R. A. J. B. S. Gottlieb, Receptors, 2001, 10, 147.

6 C. Brenner and G. Kroemer, Science, 2000, 289, 1150.

7 R. A. Eliseev, J. D. Salter, K. K. Gunter and T. E. Gunter, Biochim. Biophys. Acta, Bioenerg., 2003, 1604, 1.

8 (a) F. Gottfert, T. Pleiner, J. Heine, V. Westphal, D. Gorlich, S. J. Sahl and S. W. Hell, Proc. Natl. Acad. Sci. U. S. A., 2017, 114, 2125; (b) N. Ji, Nat. Methods, 2017, 14, 374.

9 (a) G. Song, R. Sun, J. Du, M. Chen and Y. Tian, Chem. Commun., 2017, 53, 5602; (b) P. Padmawar, X. Yao, O. Bloch, G. T. Manley and A. S. Verkman, Nat. Methods, 2005, 2, 825; (c) X. Zhou, F. Su, Y. Tian, C. Youngbull, R. H. Johnson and D. R. Meldrum, J. Am. Chem. Soc., 2011, 133, 18530; (d) B. J. Müller, S. M. Borisov and I. Klimant, Adv. Funct. Mater., 2016, 26, 7697; (e) B. Sui, X. Yue, B. Kim and K. D. Belfield, ACS Appl. Mater. Interfaces, 2015, 7, 17565; $(f)$ B. Sui, X. Yue, M. G. Tichy, T. Liu and K. D. Belfield, Eur. J. Org. Chem., 2015, 1189; $(g)$ X. Zhou, F. Su, W. Gao, Y. Tian, C. Youngbull, R. H. Johnson and D. R. Meldrum, Biomaterials, 2011, 32, 8574; (h) J. Ning and Y. Tian, Sens. Actuators, B, 2020, 307, 127659.

10 S. K. Yao, Y. Qian, Z. Q. Qi, C. G. Lu and Y. P. Cui, New J. Chem., 2017, 41, 13495.

11 P. Padmawar, X. Yao, O. Bloch, G. T. Manley and A. S. Verkman, Nat. Methods, 2005, 2, 825.

12 X. Kong, F. Su, L. Zhang, J. Yaron, F. Lee, Z. Shi, Y. Tian and D. R. Meldrum, Angew. Chem., Int. Ed., 2015, 54, 12053.

13 (a) J. Li and K. Pu, Chem. Soc. Rev., 2019, 48, 38; (b) D. Wu, L. Chen, W. Lee, G. Ko, J. Yin and J. Yoon, Coord. Chem. Rev., 2018, 354, 74; (c) G. Hong, A. L. Antaris and H. Dai, Nat. Biomed. Eng., 2017, 1.

14 (a) W. Namkung, P. Padmawar, A. D. Mills and A. S. Verkman, J. Am. Chem. Soc., 2008, 130, 7794; (b) M. Baruah, W. Qin, R. A. Vallee, D. Beljonne, T. Rohand, W. Dehaen and N. Boens, Org. Lett., 2005, 7, 4377.

15 H. He, M. A. Mortellaro, M. J. P. Leiner, R. J. Fraatz and J. K. Tusa, J. Am. Chem. Soc., 2003, 125, 1468.

16 R. D. Carpenter and A. S. Verkman, Eur. J. Org. Chem., 2011, 1242.

17 H. M. D. Bandara, Z. Hua, M. Zhang, S. M. Pauff, S. C. Miller, E. A. C. Davie and W. R. Kobertz, J. Org. Chem., 2017, 82, 8199.

18 (a) J. Li, D. Yim, W. D. Jang and J. Yoon, Chem. Soc. Rev., 2017, 46, 2437; (b) J. Yin, Y. Hu and J. Yoon, Chem. Soc. Rev., 2015, 44, 4619.

19 W. Xu, Z. Zeng, J. H. Jiang, Y. T. Chang and L. J. A. C. Yuan, Angew. Chem., Int. Ed., 2016, 55, 13658.

20 S. R. D’Mello, C. Galli, T. Ciotti and P. Calissano, Proc. Natl. Acad. Sci. U. S. A., 1993, 90, 10989.

21 C. C. Vu, C. D. Bortner and J. A. Cidlowski, J. Biol. Chem., 2001, 276, 37602 .

22 S. R. D'Mello, C. Galli and T. Ciotti, Proc. Natl. Acad. Sci. U. S. A., 1993, 90, 10989. 